\title{
SOME FUNCTIONAL EQUATIONS IN BANACH ALGEBRAS AND AN APPLICATION
}

\author{
J. VUKMAN
}

\begin{abstract}
In this paper some results concerning certain functional equations in complex Banach algebras are presented. One of these results is used to prove an abstract generalization of the classical Jordan-Neumann characterization of pre-Hilbert space.
\end{abstract}

This paper is a continuation of our earlier work $[6,7]$. Throughout this paper all Banach algebras and vector spaces are over the complex field $\mathbf{C}$. Our terminology and notation will be the same as in [7]. For Banach algebras and Banach $*$-algebras we refer to [1 and 4]. Our first few results characterize some additive functions.

THEOREM 1. Let $A$ be a Banach *-algebra with identity $e$. Let $\lambda$ and $\mu$ be automorphisms or antiautomorphisms (i.e. $\lambda(a b)=\lambda(b) \lambda(a)$ ) of $A$ (any combination is allowed). If $f: A \rightarrow A$ is an additive function such that $f(a)=\lambda(a) f\left(a^{-1}\right) \mu(a)$ for all normal invertible elements a of $A$, then $2 f(b)=\lambda(b) f(e)+f(e) \mu(b)$ for all $b \in A$.

ProOF. Let us first assume that for the function $f$

$$
f(e)=0
$$

holds and let us prove that in this case

$$
f(a)=0
$$

is fulfilled for all $a \in A$. Since $f$ is by the assumption additive, (2) will be proved if we prove that (2) holds for all normal elements. Therefore let $a \in A$ be an arbitrary normal element. One can choose rational numbers $p$ and $q$ such that $b^{-1}$ and $(e-b)^{-1}$ exist, where $b=p e+q a$. Hence $f(a)=0$ will be proved if we prove that $f(b)=0$. Now according to the requirements of the theorem and (1) we have

$$
\begin{aligned}
f(b) & =\lambda(b) f\left(b^{-1}\right) \mu(b)=\lambda(b) f\left(b^{-1}(e-b)\right) \mu(b) \\
& \left.=\lambda(b) \lambda\left(b^{-1}(e-b)\right) f(e-b)^{-1} b\right) \mu\left(b^{-1}(e-b)\right) \mu(b) \\
& =\lambda(e-b) f\left((e-b)^{-1}-e\right) \mu(e-b) \\
& =\lambda(e-b) \lambda\left((e-b)^{-1}\right) f(e-b) \mu\left((e-b)^{-1}\right) \mu(e-b) \\
& =-f(b) .
\end{aligned}
$$

Received by the editors September 26, 1985 and, in revised form, March 25, 1986. Presented to the 24th International Symposium on Functional Equations, South Hadley, Massachusetts, U.S.A., August 12-20, 1986.

1980 Mathematics Subject Classification (1985 Revision). Primary 46H05, 46K05, 39B70.

Key words and phrases. Banach algebra, Banach *-algebra, vector space, module, additive function, automorphism, antiautomorphism, pre-Hilbert space.

This research was supported by the research Council of Slovenia.

(C) 1987 American Mathematical Society $0002-9939 / 87 \$ 1.00+\$ .25$ per page 
Hence $f(b)=-f(b)$ which implies $f(a)=0$ for an arbitrary normal element $a$ and so (2) is proved. Let us now prove the theorem in full generality. For this purpose we introduce a function $g: A \rightarrow A$ defined by

$$
g(a)=f(a)-\frac{1}{2}(\lambda(a) f(e)+f(e) \mu(a)),
$$

where $f, \lambda$, and $\mu$ are such that the requirements of the theorem are fulfilled. It is obvious that $g$ is additive. A simple calculation shows that $g(a)=\lambda(a) g\left(a^{-1}\right) \mu(a)$ holds for all normal invertible elements $a \in A$. Since $g(e)=0$ we have $g(a)=0$ for all $a \in A$. In other words

$$
f(a)=\frac{1}{2}(\lambda(a) f(e)+f(e) \mu(a)) .
$$

The proof of the theorem is complete.

Using a similar approach as in the proof of Theorem 1 one can prove the following result.

THEOREM 2. Let $A$ be a Banach *-algebra with identity $e$. Let $\lambda$ and $\mu$ be automorphisms or antiautomorphisms of $A$ (any combination is allowed) such that $\lambda(a) \mu(a)=\mu(a) \lambda(a)$ for all normal invertible elements $a$ of $A$. If $f: A \rightarrow A$ is an additive function such that $f(a)=\lambda(a) \mu(a) f\left(a^{-1}\right)$ for all normal invertible elements $a$ of $A$, then $2 f(b)=(\lambda(b)+\mu(b)) f(e)$ for all $b \in A$.

One can now state two theorems in the spirit of Theorem 1 and Theorem 2 for arbitrary Banach algebras. More precisely, using a similar approach as in the proof of Theorem 1 one can prove the following two results.

THEOREM 3. Let $A$ be a Banach algebra with identity $e$. Let $\lambda$ and $\mu$ be automorphisms or antiautomorphisms (any combination is allowed). If $f: A \rightarrow A$ is an additive function such that $f(a)=\lambda(a) f\left(a^{-1}\right) \mu(a)$ for all invertible elements $a$ of $A$, then $2 f(b)=\lambda(b) f(e)+f(e) \mu(b)$ holds for all $b \in A$.

THEOREM 4. Let $A$ be a Banach algebra with identity $e$. Let $\lambda$ and $\mu$ be automorphisms or antiautomorphisms (any combination is allowed) such that $\lambda(a) \mu(a)$ $=\mu(a) \lambda(a)$ for all invertible elements $a \in A$. If $f: A \rightarrow A$ is an additive function such that $f(a)=\lambda(a) \mu(a) f\left(a^{-1}\right)$ for all invertible elements $a$ of $A$, then $2 f(b)=(\lambda(b)+\mu(b)) f(e)$ holds for all $b \in A$.

The following corollaries are immediate consequences of the theorems above (by appropriately choosing $\lambda$ and $\mu$ ).

COROllary 1. Let $A$ be a Banach *-algebra with identity e and let $f: A \rightarrow A$ be an additive function. The following statements are fulfilled.

$1^{\circ}$ If $f(a)=a f\left(a^{-1}\right) a^{*}$ holds for all normal invertible elements a of $A$, then $f$ is of the form $2 f(b)=b f(e)+f(e) b^{*}$.

$2^{\circ}$ If $f(a)=a f\left(a^{-1}\right) a$ holds for all normal invertible elements a of $A$, then $f$ is of the form $2 f(b)=b f(e)+f(e) b$.

$3^{\circ}$ If $f(a)=a^{2} f\left(a^{-1}\right)$ holds for all normal invertible elements a of $A$, then $f$ is of the form $f(b)=b f(e)$.

$4^{\circ}$ If $f(a)=a^{*} a f\left(a^{-1}\right)$ holds for all normal invertible elements a of $A$, then $f$ is of the form $f(b)=h f(e)$, where $b=h+i k$ and $h$ and $k$ are hermitian.

COROLlary 2. Let $A$ be a Banach algebra with identity $e$ and let $f: A \rightarrow A$ be an additive function. The following statements are fulfilled. 
$1^{\circ}$ If $f(a)=a f\left(a^{-1}\right) a$ holds for all invertible elements a of $A$, then $f$ is of the form $2 f(b)=b f(e)+f(e) b$.

$2^{\circ}$ If $f(a)=a^{2} f\left(a^{-1}\right)$ holds for all invertible elements $a$ of $A$, then $f$ is of the form $f(b)=b f(e)$.

It should be mentioned that the results included in the corollaries above have been proved in our earlier paper [7] under a somewhat more complicated approach and a much stronger assumption that $A$ is a hermitian Banach *-algebra (that is each hermitian element has real spectrum).

In the continuation we present the following results.

THEOREM 5. Let $A$ be a Banach *-algebra with identity $e$ and let $f: A \rightarrow A$ be an additive function. Then the following statements are fulfilled.

$1^{\circ}$ If $f(a)=-a f\left(a^{-1}\right) a^{*}$ holds for all normal invertible elements a of $A$, then $f$ is of the form $2 i f(b)=b f(i e)-f(i e) b^{*}$.

$2^{\circ}$ If $f(a)=-a^{*} a f\left(a^{-1}\right)$ hodlds for all normal invertible elements a of $A$, then $f$ is of the form $f(b)=k f(i e)$, where $b=h+i k$ and $h$ and $k$ are hermitian.

ProOF. If we introduce a function $g: A \rightarrow A$ by the relation $g(a)=f(i a)$, then $1^{\circ}$ follows from statement $1^{\circ}$ of Corollary 1 and $2^{\circ}$ from statement $4^{\circ}$ of Corollary 1.

We conclude our discussion of additive functions with the following results.

THEOREM 6. Let $A$ be a Banach *-algebra with identity $e$ and let $f: A \rightarrow$ $A, g: A \rightarrow A$ be additive functions. Then the following statements are fulfilled.

$1^{\circ}$ If $f(a)=a g\left(a^{-1}\right) a^{*}$ holds for all normal invertible elements $a$ of $A$, then $f$ and $g$ are of the form

$$
\begin{aligned}
& 2 f(b)=b(f(e)-i f(i e))+(f(e)+i f(i e)) b^{*} \\
& 2 g(b)=b(f(e)+i f(i e))+(f(e)-i f(i e)) b^{*} .
\end{aligned}
$$

$2^{\circ}$ If $f(a)=a^{*} a g\left(a^{-1}\right)$ holds for all normal invertible elements $a$ of $A$, then $f$ and $g$ are of the form

$$
f(b)=h f(e)+k f(i e), \quad g(b)=h f(e)-k f(i e),
$$

where $b=h+i k$ and $h$ and $k$ are hermitian.

PROOF. Let us prove $1^{\circ}$. From

$$
f(a)=a g\left(a^{-1}\right) a^{*}
$$

we obtain that also

$$
g(a)=a f\left(a^{-1}\right) a^{*}
$$

for all normal invertible $a \in A$. Let us introduce $F$ and $G$ by $F(a)=f(a)+g(a)$, $G(a)=f(a)-g(a) . \quad F$ and $G$ are obviously additive and from (3) and (4) one obtains easily that $F(a)=a F\left(a^{-1}\right) a^{*}$ and that $G(a)=-a G\left(a^{-1}\right) a^{*}$ holds for all normal invertible $a \in A$. Hence according to statement $1^{\circ}$ of Corollary 1 and statement $1^{\circ}$ of Theorem 5 we have

$$
2 F(a)=a F(e)+F(e) a^{*}, \quad 2 i G(a)=a G(i e)-G(i e) a^{*}
$$

for all $a \in A$. Since $g(e)=f(e)$ and $g(i e)=-f(i e)$, it follows that $f(a)+g(a)=$ $a f(e)+f(e) a^{*}$ and $f(a)-g(a)=-a i f(i e)+i f(i e) a^{*}$ for all $a \in A$. This proves 
$1^{\circ}$. Similarly one can prove that $2^{\circ}$ follows from statement $4^{\circ}$ of Corollary 1 and statement $2^{\circ}$ of Theorem 5 .

This completes our discussion of additive functions. One may also use statement $1^{\circ}$ of Corollary 1 to generalize the well-known Jordan-Neumann characterization of pre-Hilbert space. The rest of our paper does this.

THEOREM 7. Let $A$ be a Banach *-algebra with identity $e$ and let $X$ be a vector space which is also a unitary left $A$-module. Suppose there exists a mapping $Q: X \rightarrow$ $A$ with the properties

(i) $Q(x+y)+Q(x-y)=2 Q(x)+2 Q(y)$ for all pairs $x, y \in X$,

(ii) $Q(a x)=a Q(x) a^{*}$ for all $x \in X$ and all normal invertible $a$ of $A$.

Under these conditions for the mapping $B(\cdot, \cdot): X \times X \rightarrow A$ defined by the relation

$$
B(x, y)=\frac{1}{4}(Q(x+y)-Q(x-y))+\frac{i}{4}(Q(x+i y)-Q(x-i y))
$$

the following statements are fulfilled.

$1^{\circ} B(\cdot, \cdot)$ is additive in both arguments.

$2^{\circ} B(a x, y)=a B(x, y), B(x, a y)=B(x, y) a^{*}$ for all pairs $x, y \in X$ and all $a \in A$.

$$
3^{\circ} Q(x)=B(x, x) \text { for all } x \in X \text {. }
$$

The result above was proved in [7] under the stronger assumption that $A$ is a hermitian Banach *-algebra (see also [6]). If $A$ is the complex number field, then Theorem 7 reduces to S. Kurepa's extension of Jordan-Neumann theorem (see [2, 3] and also [5]).

The proof of Theorem 7 we shall omit since it goes through in the same way as in [7] with the only exception that for the proof of statement $2^{\circ}$ one has to use statement $1^{\circ}$ of Corollary 1 instead of Theorem 1.3 in [7].

ACKNOWLEDGMENTS. I wish to express my sincere thanks to Professor P. Vrbová for helpful conversations. I am also indebted to the referee for many suggestions.

\section{REFERENCES}

1. F. F. Bonsall and J. Duncan, Complete normed algebras, Springer-Verlag, Berlin and New York, 1973.

2. S. Kurepa, The Cauchy functional equation and scalar product in vector spaces, Glas. Mat. Fiz.-Astr. 19 (1964), 23-36.

3. __ Quadratic and sesquilinear functionals, Glas. Mat. Fiz.-Astr. 20 (1965), 79-92.

4. C. E. Rickart, Banach algebras, Kreiger, Hunington, N.Y., 1974.

5. P. Vrbová, Quadratic functionals and bilinear forms, Casopis Pést. Mat. 98 (1973), 159-161.

6. J. Vukman, A result concerning additive functions in hermitian Banach *-algebras and an application, Proc. Amer. Math. Soc. 91 (1984), 367-372.

7. Some results concerning the Cauchy functional equation in certain Banach algebras, Bull. Austral. Math. Soc. 31 (1985), 137-144.

Department of Mathematics, University of MaRibor, 62000 MaRibor, YuGOSLAVIA 